\title{
Non-invasive ventilation in the elderly - never too late!
}

\author{
Leonor Mendonça Almeida', Maria Jacob', Mafalda van Zeller²,3,4, Tiago Pinto ${ }^{2,4}$, Miguel Gonçalves ${ }^{1,2,3,4,5}$, \\ Marta Drummond $2,3,4$ \\ ${ }^{1}$ Pulmonology Department, Centro Hospitalar Universitário São João, Porto; ${ }^{2}$ Sleep and Non-invasive Ventilation Unit, \\ Centro Hospitalar Universitário São João, Porto; ${ }^{3}$ Faculty of Medicine, University of Porto; ${ }^{4} i 3 S$ - Institute for Research \\ and Innovation in Health, Porto; ${ }^{5}$ Intensive Care Medicine Department, Centro Hospitalar e Universitário São João, \\ Porto, Portugal
}

\begin{abstract}
Dear Editor,
Practising evidence-based medicine in an ageing population is challenging. Nevertheless, using age as a diagnostic or therapeutic procedure contraindication is less and less common. Domiciliary non-invasive ventilation (NIV) in chronic respiratory failure
\end{abstract}

Correspondence: Leonor Furtado de Mendonça e Almeida Cunha, Pulmonology Department, Centro Hospitalar e Universitário de São João, Alameda Professor Hernâni Monteiro, 4200-319 Porto, Portugal. Tel. +351.934.474-855.

E-mail: leonor.fma@gmail.com

Key words: Non-invasive ventilation; elderly; ageing.

Contributions: LMA, conception and design, data collection, analysis, interpretation of data, manuscript drafting, finalizing the version to be published; MJ, data collection, statistical analysis, substantial contribution to interpretation of data, draft revision; MvZ, conception and design, contribution to analysis and interpretation of data, draft revision; TP, contribution to analysis and interpretation of data, draft revision; MRG, contribution to analysis and interpretation of data, draft revision; MD, conception and design, contribution to analysis and interpretation of data, draft revision.

Conflict of interest: Dr. Mendonça Almeida reports non-financial support from Vitalaire, outside the submitted work; Dr. M. Jacob reports non-financial support from Gasoxmed, outside the submitted work; Dr. R. Gonçalves reports personal fees from Nippon gases, personal fees from Linde Healthcare, personal fees from ResMed, personal fees from Philips Respironics, personal fees from B\&D Eletromedical, outside the submitted work; Dr. M. Drummond reports personal fees from Vitalaire, non-financial support from D'Ar Saúde, non-financial support from Gasoxmed, outside the submitted work. The other authors declare no conflict of interest.

Ethics approval: The study protocol was approved by the Local Ethics Committee.

Received for publication: 3 February 2021

Accepted for publication: 24 February 2021.

COpyright: the Author(s), 2021

Licensee PAGEPress, Italy

Monaldi Archives for Chest Disease 2021; $91: 1796$

doi: 10.4081/monaldi.2021.1796

This article is distributed under the terms of the Creative Commons Attribution Noncommercial License (by-nc 4.0) which permits any noncommercial use, distribution, and reproduction in any medium, provided the original author(s) and source are credited. patients has been largely used; however, data from older people is scarce. The latest European Respiratory Society guidelines for long-term home NIV for management of chronic obstructive pulmonary disease (COPD) point out the need for more research in the elderly since the initial trials excluded patients older than 65 and age is no longer a contraindication, but the mean age in trials is around 65 years old. Our centre has a significant proportion of older patients under domiciliary NIV, so we felt the need to share our experience to increase the evidence in the ageing population.

We performed a longitudinal retrospective study of patients with $\geq 75$ years old under NIV in one year in a chronic setting. Patients were adapted in an NIV-lab, using thoracoabdominal bands, peripheral oxygen saturation and transcutaneous carbon dioxide ( $\mathrm{TcCO} 2)$ monitoring. Patients were ambulatory or were already admitted as an inpatient, and home NIV was decided during admission. Evaluation of efficacy and adherence were performed at the first month and one year after. Descriptive data were expressed as frequencies and percentages for qualitative and ordinal variables, and as the mean and standard deviation (SD) for quantitative data. Student's $t$-test or Wilcoxon test were used to comparing quantitative variables and Chi-square test or Fisher exact test to categorical variables (SPSS v. 25.0).

There were 261 in-lab NIV titrations in one year, and 82 (31.4\%) performed in patients older than 75 were included in this study. The majority were male $(43,52.4 \%)$, with a mean age of $82.1 \pm 5$ years old. Treatment indications included obstructive sleep apnoea with nocturnal hypoventilation (23, 28\%), COPD (17, $20.7 \%$ ) and obesity hypoventilation syndrome (16, 19.5\%). Most patients $(72 \%)$ presented with hypercapnic respiratory failure, $3.7 \%$ were hypoxemic, and $20.4 \%$ without respiratory failure. Patients' characteristics and NIV related information are summarised in Table 1.

The adherence at the first month of treatment was adequate and remained (93.4\%/days with 7.46 hours/night at first month and $89 \%$ /days with $8 \mathrm{~h} 16 /$ night at 12 months, $\mathrm{p}=0.696)$. Fourteen patients $(17.1 \%)$ suspended NIV during follow-up (Table 1). One patient under adaptative servo-ventilation had a reduction in left ventricle ejection fraction and, therefore, NIV was discontinued. Patients who suspended NIV due to intolerance or refusal were not ventilated with higher pressures than other patients (mean IPAP $19 \pm 4.4$ vs $19.2 \pm 4.7, \mathrm{p}=0.905$; mean EPAP $7 \pm 2.1$ vs $7.5 \pm 2.4$, $\mathrm{p}=0.493$; support pressure $12 \pm 4.9 v s 11.7 \pm 4.6, \mathrm{p}=0.858$ ) and none was secondary to interface related side effects.

Regarding treatment efficacy, oxygen saturation $\left(\mathrm{SpO}_{2}\right)$ increased, and $\mathrm{CO}_{2}$ arterial pressure $\left(\mathrm{PaCo}_{2}\right)$ decreased significantly at the end of the first month $\left(\mathrm{SpO}_{2} 90.7 \pm 3.9 \%\right.$ in the baseline, $94.4 \pm 2.9$ in the first month, $\mathrm{p}<0.001 ; \mathrm{PaCO}_{2} 51.5 \pm 8.8 \mathrm{mmHg}$ 
Table 1. Complete cohort characteristics and comparison between patients under and over 85 years of age.

\begin{tabular}{|c|c|c|c|c|}
\hline Characteristics & Total patients & $<85$ years-old & $\geq 85$ years-old & p \\
\hline $\begin{array}{l}\text { Sex }(n, \%) \\
\text { Male } \\
\text { Female }\end{array}$ & $\begin{array}{l}43,52.4 \% \\
39,47.6 \%\end{array}$ & $\begin{array}{l}32,59.3 \% \\
22,40.7 \%\end{array}$ & $\begin{array}{l}11,39.3 \% \\
17,60.7 \%\end{array}$ & $0.086^{*}$ \\
\hline $\begin{array}{l}\text { Age }(\text { mean } \pm \text { SD }) \\
\geq 75,<85 \text { years old }(n, \%) \\
\geq 85 \text { years old }(n, \%)\end{array}$ & $\begin{array}{l}82.1 \pm 5.2 \\
54,65.9 \% \\
28,34.1 \%\end{array}$ & & & \\
\hline Obesity (n, \%) & $32,39 \%$ & $21,38 \%$ & $11,39 \%$ & $0.972 *$ \\
\hline Heart failure (n, \%) & $39,47.6 \%$ & $24,44.4 \%$ & $15,53.6 \%$ & $0.433^{*}$ \\
\hline $\begin{array}{l}\text { NIV indication (n, \%) } \\
\text { OSAS with hypoventilation } \\
\text { COPD } \\
\text { Overlap COPD-OSAS } \\
\text { OHS } \\
\text { Neuromuscular disease } \\
\text { Chest-wall disease } \\
\text { ILD }\end{array}$ & $\begin{array}{c}23,28 \% \\
17,20.7 \% \\
16,19.5 \% \\
16,19.5 \% \\
5,6.1 \% \\
4,4.9 \% \\
1,1.2 \%\end{array}$ & $\begin{array}{c}16,29.6 \% \\
13,24.1 \% \\
10,18.5 \% \\
7,13.0 \% \\
4,7.4 \% \\
3,5.6 \% \\
1,1.8 \%\end{array}$ & $\begin{array}{c}7,25 \% \\
4,14.3 \% \\
6,21.4 \% \\
9,32.1 \% \\
1,3.6 \% \\
1,3.6 \% \\
0,0 \%\end{array}$ & $0.462^{*}$ \\
\hline $\begin{array}{l}\text { Oxygen }(\mathrm{n}, \%) \\
\text { LTOT } \\
\text { AOT }\end{array}$ & $\begin{array}{c}24,29.3 \% \\
1,1.2 \%\end{array}$ & $\begin{array}{l}17,31.5 \% \\
0,0 \%\end{array}$ & $\begin{array}{l}7,28 \% \\
1,3.6 \%\end{array}$ & $0.330^{*}$ \\
\hline NIV adaptation setting (n, \%) & $18,22 \%$ & $11,20.4 \%$ & $7,25 \%$ & $0.631^{*}$ \\
\hline $\begin{array}{l}\text { Inpatient/post-exacerbation } \\
\text { Ambulatory }\end{array}$ & $64,78 \%$ & $43,79.6 \%$ & $21,75 \%$ & \\
\hline $\begin{array}{l}\text { Mode } \\
\text { Bilevel ST (n, \%) } \\
\text { Bilevel S (n, \%) } \\
\text { Auto-bilevel (n, \%) } \\
\text { AVAPS (n, \%) } \\
\text { ASV (n, \%) }\end{array}$ & $\begin{array}{c}75,91.5 \% \\
2,2.4 \% \\
1,1.2 \% \\
1,1.2 \% \\
3,3.7 \%\end{array}$ & $\begin{array}{c}48,88.9 \% \\
2,3.7 \% \\
1,1.8 \% \\
0,0 \% \\
3,5.6 \%\end{array}$ & $\begin{array}{c}27,96.4 \% \\
0,0 \% \\
0,0 \% \\
1,3.6 \% \\
0,0 \%\end{array}$ & $0.272^{*}$ \\
\hline $\begin{array}{l}\text { Settings } \\
\text { IPAP }(\text { mean } \pm \text { SD) } \\
\text { EPAP }(\text { mean } \pm \text { SD) } \\
\text { RR }(\text { mean } \pm \text { SD) }\end{array}$ & $\begin{array}{l}19.2 \pm 4.4 \\
7.47 \pm 2.4 \\
16.1 \pm 1.9\end{array}$ & $\begin{array}{l}18.6 \pm 4.3 \\
7.41 \pm 2.4 \\
11.2 \pm 5.0\end{array}$ & $\begin{array}{l}20.3 \pm 4.4 \\
7.57 \pm 2.4 \\
12.7 \pm 3.6\end{array}$ & $\begin{array}{l}0.103^{¥} \\
0.778^{\ddagger} \\
0.115^{¥}\end{array}$ \\
\hline $\begin{array}{l}\text { Adherence } \\
1^{\text {st }} \text { month } \\
\% \text { days (mean } \pm \text { SD) } \\
\text { Hours/night }(\text { mean } \pm \text { SD) } \\
12 \text { months } \\
\% \text { days (mean } \pm \text { SD) } \\
\text { Hours/night (mean } \pm \text { SD) }\end{array}$ & $\begin{array}{c}93.4 \pm 20.2 \\
7: 46 \pm 2: 40 \\
89 \pm 26.6 \\
7: 59 \pm 2: 37\end{array}$ & $\begin{array}{l}80.5 \pm 34.0 \\
7: 01 \pm 2: 35 \\
81.6 \pm 33.3 \\
7: 26 \pm 2: 57\end{array}$ & $\begin{array}{l}91.1 \pm 15.7 \\
7: 01 \pm 2: 20 \\
88.8 \pm 26.8 \\
7: 34 \pm 1: 49\end{array}$ & $\begin{array}{l}0.068^{\ddagger} \\
0.997^{\ddagger} \\
0.454^{¥} \\
0.867^{¥}\end{array}$ \\
\hline $\begin{array}{l}\mathrm{PaCO}_{2}(\mathrm{mmHg}, \text { mean } \pm \mathrm{SD}) \\
\text { Baseline } \\
1^{\text {st }} \text { month } \\
12 \text { months }\end{array}$ & $\begin{array}{l}51 \pm 10.5 \\
44.6 \pm 6.9 \\
42.3 \pm 7.0\end{array}$ & $\begin{array}{c}49.5 \pm 9.4 \\
43.7 \pm 6.2 \\
41.6 \pm 6.6\end{array}$ & $\begin{array}{c}53 \pm 8.1 \\
46.2 \pm 7.9 \\
43.5 \pm 7.6\end{array}$ & $\begin{array}{l}0.101^{¥} \\
0.186^{¥} \\
0.434^{¥}\end{array}$ \\
\hline $\begin{array}{l}\mathrm{SpO}_{2}(\%, \text { mean } \pm \mathrm{SD}) \\
\text { Baseline } \\
1^{\text {st }} \text { month } \\
12 \text { months }\end{array}$ & $\begin{array}{l}90.4 \pm 4.5 \\
94.1 \pm 3.2 \\
94.6 \pm 2.5\end{array}$ & $\begin{array}{l}90.7 \pm 4.4 \\
93.9 \pm 3.3 \\
94.4 \pm 2.7\end{array}$ & $\begin{array}{l}90.9 \pm 3.1 \\
95.1 \pm 1.5 \\
95.6 \pm 1.6\end{array}$ & $\begin{array}{l}0.849^{\#} \\
0.093^{\#} \\
0.119^{\#}\end{array}$ \\
\hline $\begin{array}{l}\text { NIV suspension (n, \%) } \\
\text { Refusal } \\
\text { Intolerance to pressure } \\
\text { Poor adherence } \\
\text { LVEF < } 45 \%\end{array}$ & $\begin{array}{c}14,17.1 \% \\
6 \\
5 \\
2 \\
1\end{array}$ & $\begin{array}{c}10,18.5 \% \\
5 \\
4 \\
0 \\
1\end{array}$ & $\begin{array}{c}4,14.3 \% \\
1 \\
1 \\
2 \\
0\end{array}$ & $0.762^{*}$ \\
\hline $\begin{array}{l}\text { Admissions for RF (n, \%) } \\
\text { Before NIV } \\
\text { 0/year } \\
1-2 / \text { year } \\
>2 \text { /year } \\
1^{\text {st } y e a r ~ p o s t ~ N I V ~}\end{array}$ & $\begin{array}{c}39,47.6 \% \\
35,42.7 \% \\
8,9.7 \%\end{array}$ & $\begin{array}{c}28,51.9 \% \\
20,37.0 \% \\
6,1.1 \%\end{array}$ & $\begin{array}{c}11,39.3 \% \\
15,53.6 \% \\
2,7.1 \%\end{array}$ & $0.352^{*}$ \\
\hline $\begin{array}{l}0 / \text { year } \\
0 \text {-1/year }\end{array}$ & $\begin{array}{l}59,71.9 \% \\
21,25.6 \%\end{array}$ & $\begin{array}{l}42,77.8 \% \\
11,20.4 \%\end{array}$ & $\begin{array}{l}17,60.7 \% \\
10,35.7 \%\end{array}$ & $0.264^{*}$ \\
\hline Mortality in $1^{\text {st }}$ year $(n, \%)$ & $16,19.5 \%$ & $9,15.8 \%$ & $7,25 \%$ & $0.367^{*}$ \\
\hline
\end{tabular}

${ }^{*}$ Chi-square,test or Fisher exact test; "independent samples $t$-test; AOT, ambulatory oxygen therapy; ASV, adaptative servoventilation; AVAPS, average volume, assured pressure support; EPAP, expiratory positive airway pressure; ILD, interstitial lung disease; IPAP, inspiratory positive airway pressure; LTOT, long term oxygen therapy; LVEF, left ventricle ejection fraction; OHS, obesity hypoventilation syndrome; OSAS, obstructive sleep apnoea syndrome; RR, respiratory rate; SD, standard deviation; ST, spontaneous/timed; S, spontaneous. 

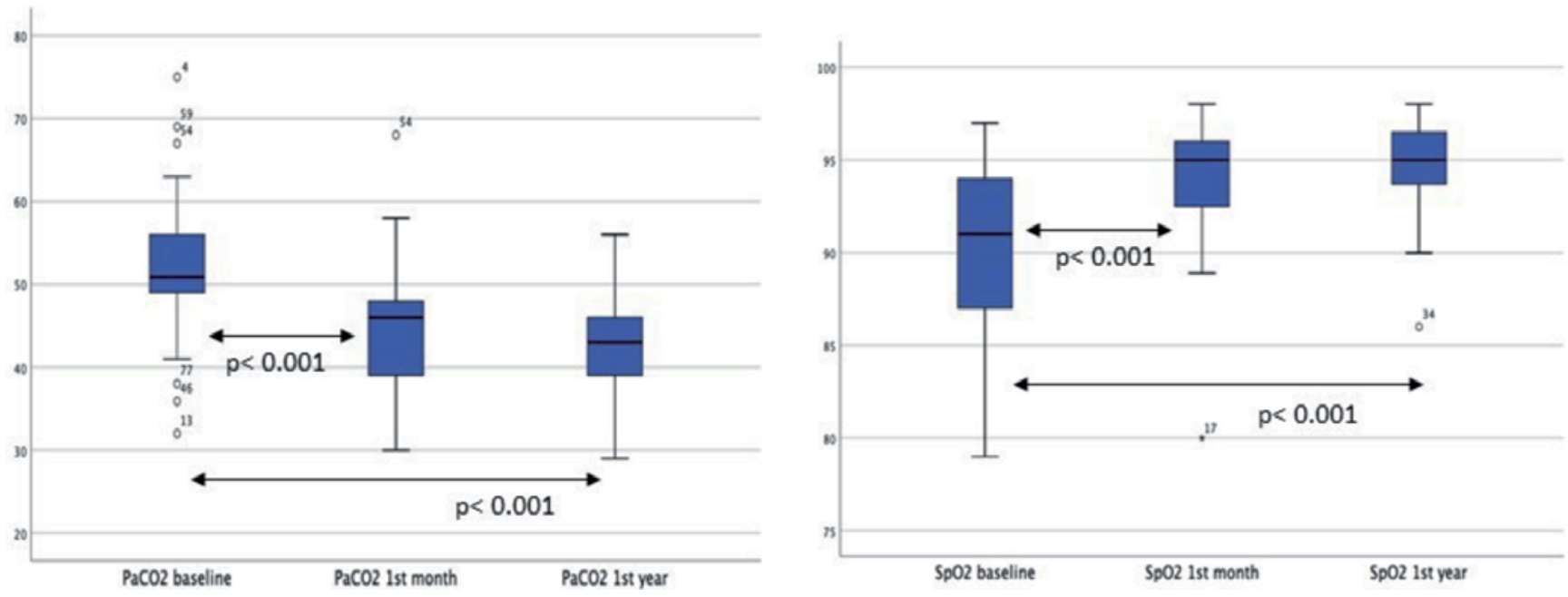

Figure 1. Variation in carbon dioxide arterial pressure $\left(\mathrm{PaCO}_{2}\right)$ and oxygen peripheral saturation $\left(\mathrm{SpO}_{2}\right)$.

in the baseline vs $44.6 \pm 6.9 \mathrm{mmHg}$ in the first month, $\mathrm{p}<0.001$ ). These values were maintained at 12 months with significant variation from baseline $\left(\mathrm{SpO}_{2} 94.6 \pm 2.5 \%, \mathrm{p}<0.001 ; \mathrm{PaCO}_{2} 42.3 \pm 7\right.$ $\mathrm{mmHg}, \mathrm{p}<0.001$ ) (Figure 1).

A reduction in mean hospitalisations for respiratory failure compared with the previous year was also observed $(1.02 \pm 1.6 \mathrm{vs}$ $0.29 \pm 0.51, \mathrm{p}<0.001)$. Sixteen patients died in the first year (19.5\%). Mortality was associated with hospitalisation $(38.1 \%$ mortality in patients with at least one hospitalisation in the followup year vs $10.2 \%$ mortality in patients without admissions, $\mathrm{p}<0.001)$.

When patients were divided into two age groups, under and over 85 years old, there were no significant differences between groups in none of the analysed variables (Table 1).

Our data outlines that home NIV is a well-tolerated and effective treatment in elderly patients, correcting hypercapnia and reducing hospitalisations.

NIV in the acute setting has well-established indications, and its feasibility in the elderly population has been shown $[1,2]$. In chronic patients, some indications, such COPD, are still lacking robust evidence, despite the physiological plausibility and disseminated prescription across Europe [3]. This paucity of evidence is even more noteworthy in the elderly. Few retrospective studies address the efficacy and tolerability of domiciliary NIV in elderly patients [4,5]; however, the mean age in these studies is under 80 . In our study, the mean age is 82 , and $34 \%$ of patients were above 85; nevertheless, the older group of patients still benefited from this therapy, having similar reductions in $\mathrm{CO} 2$ and hospitalisations. The described compliance was in line or slightly better than previ- ously reported, both in general and elder population [4,5]. This study has several limitations, including its retrospective design. On the other hand, this is a heterogeneous cohort regarding the indication to initiate NIV treatment and further studies in elderly in NIV across different indications are needed. Nonetheless, since this cohort has a significant proportion of ancient patients benefiting, tolerating, and complying very well with home NIV, the authors considered it was relevant to share these data with the scientific community.

\section{References}

1. Nava S, Grassi M, Fanfulla F, et al. Non-invasive ventilation in elderly patients with acute hypercapnic respiratory failure: a randomised controlled trial. Age Ageing 2011;40:444-50.

2. Montoneri G, Noto P, Trovato FM, et al. Outcomes of noninvasive ventilation in 'very old' patients with acute respiratory failure: a retrospective study. Emerg Med J 2019;36:303-5.

3. Crimi C, Noto A, Princi P, et al. Domiciliary non-invasive ventilation in COPD: An international survey of indications and practices. COPD 2016;13:483-90.

4. Farrero E, Prats E, Manresa F, Escarrabill J. Outcome of noninvasive domiciliary ventilation in elderly patients. Respir Med 2007;101:1068-73.

5. Crespo A, Muñoz X, Torres F, et al. Non-invasive home mechanical ventilation in elderly patients. Gerontology 2010;56:150-6. 